\title{
E-LEARNING COMO ESTRATEGIA DE INTERNACIONALIZACIÓN DE LA EDUCACIÓN SUPERIOR
}

\author{
Dr. David Roldán Martínez \\ darolmar@upvnet.upv.es \\ Dr. Antonio Hervás Jorge \\ ahervas@mat.upv.es \\ Instituto de Matemática Multidisciplinar \\ Universidad Politécnica de Valencia
}

\section{RESUMEN}

Este trabajo plantea un estudio de la internacionalización de la Educación Superior desde el punto de vista de las Universidades y del e-learning como estrategia competitiva e introduce un procedimiento sistemático de análisis que pretende asistir a las instituciones de Educación Superior en la elaboración de sus planes estratégicos y tácticos en cuanto a la formación on-line se refiere, siempre con el objetivo de conseguir una posición y una ventaja competitivas sólidas y estables.

\section{PALABRAS CLAVE:}

Educación superior, internacionalización, estrategia, análisis estratégico, cadena de valor, sistema de valor, modelo de negocio, competitividad, e-learning.

\section{ABSTRACT}

This work analyzes Higher Education internationalization from the point of view of Universities and focusing at the use of e-learning as competitive strategy. It also introduces a systematic analysis process so that Higher Education Institution can use it to elaborate its strategic and tactical plans in relation with e-learning, always trying to reach both solid and stable competitive position and advantage.

\section{KEY WORDS:}

Higher education, internationalization, strategy, strategic analysis, value chain, value system, business model, competition, e-learning. 


\section{LA UNIVERSIDAD SE TRANSFORMA}

La evolución de la Educación Superior en los últimos años se caracteriza por la adopción de parámetros de eficiencia, en la reducción de las estructuras organizativas y en fomento de procesos de descentralización en la ejecución de la actividad.

Los agentes de cambio que están llevando a la transformación de las Universidades y a la adopción de procesos de internacionalización en las mismas, pueden agruparse en cinco grupos:

- La evolución de la demanda de enseñanza universitaria, que se concreta en la potenciación de la demanda social de ampliación del acceso a dicha formación, el incremento de la demanda de formación universitaria por parte de la población adulta.

- La adaptación de la oferta formativa a los nuevos requerimientos de capacidad y habilidades de los trabajadores para atender las exigencias de actualización y reciclaje permanente de las habilidades de los trabajadores, características estas de la economía del conocimiento.

- La demanda social de una mayor transparencia informativa sobre los resultados de la gestión de la actividad Universitaria y de su efecto sobre el conjunto de la Sociedad.

- La evolución de la financiación de las Universidades, caracterizada por una disminución de los recursos disponibles y por una alteración de la composición de la estructura financiera con un incremento significativo de los recursos financieros privados y de la autofinanciación en detrimento de los fondos públicos.

- Promoción de la movilidad de los integrantes de la comunidad universitaria a través de la eliminación de los obstáculos a la libre circulación de estudiantes, profesores e investigadores, favorecida por la utilización de las TIC, y que en Europa se ha materializado en el Espacio Europeo de Educación Superior.

- Establecimiento de un marco internacional para el comercio mundial de servicios educativos.

- La traslación de los modelos educativos estadounideneses, caracterizados por su orientación a mercado, a otros contextos (en nuestro caso el europeo) frente a los modelos educativos controlados por el Estado que han resultado en una menor competitividad a la hora de competir en el mercado internacional.

- La introducción de las TIC en las Universidades y sus repercusiones a todos los niveles de la actividad universitaria.

Algunos de estos impulsores pertenecen a varios de los ámbitos señalados, de hecho, resulta difícil encontrar alguno que no tenga un matiz económico.

Por todos estos motivos, las Universidades se enfrentan al reto de adaptarse a este nuevo paradigma socioeconómico. En este contexto, el empleo de las TIC en la actividad universitaria se está convirtiendo en un elemento instrumental importante para conseguir los objetivos marcados, lo que 
explica la aparición de una nueva forma de realizar la acción docente basada en el uso intensivo de las TIC: el e-learning.

\section{...Y, ¿CÓMO AFRONTAR EL CAMBIO?}

En este nuevo entorno, las Universidades deben reaccionar a través de la asunción de prácticas empresariales. En efecto, tal y como Duart y Lupiáñez (2005) señalan, esta transferencia procedente del mundo empresarial será beneficiosa siempre y cuando se sepa trasladar adecuadamente y no se deje de lado la labor social de la Universidad.

Cuando cualquier organización se plantea competir en un determinado sector de actividad, el paso previo consiste en definir claramente el producto o servicio. En estos términos, el presente trabajo trata sobre el negocio de la Educación Superior (mercado) utilizando el e-learning como soporte de la formación en red (tecnología) con el fin de que los estudiantes adquieran los conocimientos y habilidades necesarias para ofrecer acceso a recursos educativos a distancia (funciones).

Una vez definido el negocio, el paso siguiente será determinar cómo desarrollar una estrategia en el sector en el que se compite y en el que se luchará con otras organizacionescuya definición del negocio es similar. La diferencia entre el éxito y el fracaso estará, pues, no la definición del negocio, sino en su implementación o modelo de negocio, concepto éste que adquiere una vital importancia.

El objetivo último de la la construcción del modelo de negocio es evaluar la probabilidad de que una idea estratégicamente relevante para la institución sea, efectivamente viable antes de la asignación de recursos para la materialización de la misma.

El modelo de negocio unifica decisiones importantes en el ámbito empresarial desde los puntos de vista económico, operacional y estratégico. Constituye una unidad de análisis muy útil que facilita el desarrollo teórico de la puesta en marcha de una idea empresarial. La definición de un buen modelo de negocio es una tarea compleja que, sin embargo, puede esquematizarse en los siguientes procesos:

1. Analizar estratégicamente el sector con el fin de detectar oportunidades y amenazas, comprender el comportamiento del mismo e descubrir la estructura del sector así como las razones que hacen que ésta sea así.

2. Articular la proposición de valor, esto es, el valor creado para los usuarios del modelo por el producto (bien y/o servicio) ofrecido usando la tecnología.

3. Identificar un segmento del mercado, para dichos usuarios el uso de la tecnología debe ser útil y la empresa debe obtener ingresos por el ofrecimiento de la misma.

4. Definir la estructura de la cadena de valor que la organización necesita para crear y distribuir su producto y determinar los activos complementarios necesarios para apoyar la posición de la empresa 
5. Estimar la estructura de costes y los beneficios potenciales para ofrecer su producto, teniendo en cuenta la proposición de valor y la estructura de la cadena de valor elegida.

6. Describir la posición de la empresa dentro del sistema de valor, relacionando proveedores y clientes.

7. Formular la estrategia competitiva a través de la cual la empresa innovadora será más competitiva y obtendrá una ventaja sostenible.

\section{ANÁLISIS ESTRATÉGICO DEL SECTOR}

El objetivo del análisis del sector es relacionar a la organización con su entorno competitivo, determinar la estructura del sector y las razones de que sea así. Para ello, tradicionalmente se sigue el enfoque propuesto por Porter al que en este artículo se añadido una fuerza competitiva más, habida cuenta de que Europa en general y España en particular se rige por un modelo de sistema educativo regulado: el poder negociador del Gobierno (ver Figura 1).

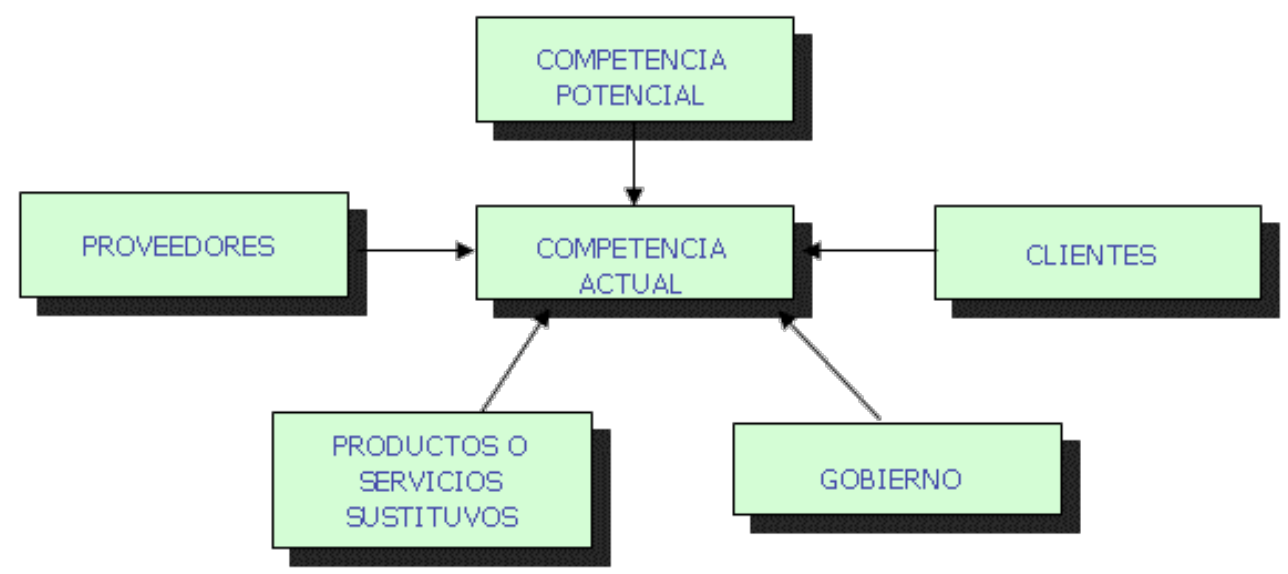

Figura 1. Modelo de fuerzas competitivas en el sector del e-learning y la Educación Superior.

Fuente: elaboración propia. 
Los competidores actuales en el sector de la Educación Superior on-line son los mismos que en el caso del formato presencial tradicional. Con el fin de agruparlos de cara a investigaciones futuras, se propone seguir una clasificación en función de un doble criterio: la intensidad de utilización del e-

\begin{tabular}{|l|l|}
\hline Tipo de Universidad & Descripción \\
\hline $\begin{array}{l}\text { Universidades de e- } \\
\text { learning }\end{array}$ & $\begin{array}{l}\text { Empleo de los sistemas de e-learning como elemento principal de la } \\
\text { docencia. }\end{array}$ \\
\hline Universidades duales & $\begin{array}{l}\text { Modelo de formación a distancia flexible y basado en TIC y } \\
\text { modelos presenciales con integración de sistemas de e-learning. }\end{array}$ \\
\hline $\begin{array}{l}\text { Universidades con un uso } \\
\text { complementario del e- } \\
\text { learning }\end{array}$ & $\begin{array}{l}\text { Modelos a distancia tradicionales pero flexibles y modelos } \\
\text { presenciales con un uso del e-learning como soporte a la } \\
\text { metodología tradicional. }\end{array}$ \\
\hline $\begin{array}{l}\text { Universidades } \\
\text { tradicionales }\end{array}$ & $\begin{array}{l}\text { Modelos a distancia y presenciales que presentan un uso residual de } \\
\text { las TIC. }\end{array}$ \\
\hline
\end{tabular}

learning en la metodología docente (ver Figura 2) y el modelo organizativo (ver Figura 3).

Fuente: elaboración propia.

Figura 2. Clasificación de las Universidades en función de su empleo del e-learning.

\begin{tabular}{|c|c|}
\hline Modelo organizativo & Descripción \\
\hline $\begin{array}{l}\text { Universidades virtuales como organizaciones } \\
\text { virtuales }\end{array}$ & $\begin{array}{l}\text { Son universidades creadas específicamente para } \\
\text { realizar una actividad de e-learning, algunas de las } \\
\text { cuales parten de modelos de universidades abiertas y } \\
\text { a distancia. Se caracterizan por disponer de un } \\
\text { modelo organizativo y pedagógico particular y } \\
\text { diferenciado del resto de universidades presenciales } \\
\text { y a distancia. }\end{array}$ \\
\hline $\begin{array}{l}\text { Universidad presencial con extensión universitaria } \\
\text { virtual }\end{array}$ & $\begin{array}{l}\text { Se trata de un modelo muy extendido en las } \\
\text { Universidades y que consiste en incorporar } \\
\text { formación virtual en cursos de postgrado y extensión } \\
\text { universitaria en instituciones educativas cuya oferta } \\
\text { de formación reglada es eminentemente presencial. }\end{array}$ \\
\hline $\begin{array}{l}\text { Universidad virtual adosada a la universidad } \\
\text { tradicional }\end{array}$ & $\begin{array}{l}\text { Se trata de universidades virtuales creadas por } \\
\text { universidades tradicionales en su mismo entorno a } \\
\text { través de espacios virtuales gestionados de forma } \\
\text { independiente pero compartiendo los elementos } \\
\text { básicos de la universidad tradicional (oferta } \\
\text { fomativa, normativa académica, servicios de } \\
\text { biblioteca, etc.). }\end{array}$ \\
\hline $\begin{array}{l}\text { Universidades virtuales como espacios virtuales } \\
\text { interuniversitarios }\end{array}$ & $\begin{array}{l}\text { Se trata una universidad virtual creada a través de un } \\
\text { consorcio de diversas universidades tradicionales y } \\
\text { se materializa en la disponibilidad de un espacio } \\
\text { virtual común en el que converge la oferta de los } \\
\text { diferentes planes de estudio. Este espacio se gestiona } \\
\text { de forma compartida pero autónoma en la } \\
\text { Universidades participantes en el consorcio. }\end{array}$ \\
\hline Modelo de intermediación & $\begin{array}{l}\text { La o las Universidades se asocian con una empresa } \\
\text { que proporciona cursos utilizando los recursos de } \\
\text { enseñanza de instituciones existentes }\end{array}$ \\
\hline Universidad Corporativa & $\begin{array}{l}\text { Unidad de negocios de una empresa que se encarga } \\
\text { de gestionar los planes de formación de los } \\
\text { empleados de la misma. }\end{array}$ \\
\hline
\end{tabular}

Fuente: elaboración propia.

Figura 3. Modelos organizativos de universidades virtuales. 
Otro de los aspectos que influyen en la competencia actual son las barreras de salida, bajo cuya denominación se agrupan todos aquellos aspectos que obligan a seguir compitiendo en el sector. En el caso del e-learning y la Educación Superior, se han identificado las siguientes barreras de salida:

- Dificultades de desinversión: activos altamente especializados y que tienen poco valor liquidativo, como es el caso de la plataforma de e-learning o laboratorios de generación de contenidos.

- Personal fijo: puede llegar a ser una barrera importante. Por esta razón, se aconseja contratar profesores a tiempo parcial y solamente tener fijos al personal administrativo.

- Cancelación de contratos con clientes: barrera importante si se imparten cursos largos. Por otro lado, dado el papel social de la Universidad es posible que la institución se vea obligada a mantener titulaciones poco rentables.

\section{Competencia potencial}

Los competidores potenciales son, básicamente, los mismos que los actuales, a los que habría que añadir las Universidades Corporativas y los consorcios educativos. La competencia potencial, entre otros aspectos, viene determinada por las barreras de entrada, que pueden definirse como el conjunto de factores de tipo económico, social e institucional que constituyen un freno o impedimento para el ingreso de nuevas empresas en el sector. Se han identificado las siguientes:

- Imagen de marca

El mercado de la Educación Superior la imagen de marca y el prestigio adquieren una doble importancia. En primer lugar, supone una barrera de entrada muy alta que frena a nuevos competidores a entrar a competir en el mercado. Por otra parte, constituye uno de los factores determinantes tanto para la financiación pública, como para la financiación privada.

Con el fin de proyectar una imagen de marca integral, sólida y consistente que permita a las instituciones tener un respaldo estratégico, las Universidades deben adoptar, entre otras, las siguientes medidas:

- Concretar su identidad corporativa, a partir de la formulación de un mensaje institucional unificado, claro, concreto y conciso que impacte en cualquier público del mundo.

- Fomentar redes académicas que potencialicen la divulgación de los resultados de trabajos académicos y de investigación.

- Fortalecer las acciones de relaciones públicas en tiempo real, para contribuir a la proyección de la imagen institucional.

- Construir vínculos estables para generar confianza con los diferentes públicos.

- Acceso privilegiado a materias primas 
Los centros sin prestigio o no relacionados, tendrán dificultades para atraer buenos profesores. Si lo consiguen, será a alto coste. En caso de que el centro no disponga de PDI de prestigio deberá saber qué busca cualquier profesor a la hora de colaborar con una institución educativa. Algunos aspectos importantes en este sentido son:

- Programas de I+D competitivos.

- Posibilidad de promocionarse entre los alumnos.

- Ser un medio para facilitar al profesor la puesta al día en los temas de su especialidad o interés.

- Resultar atractivo para su curriculum.

- Economías de escala

Las economías de escala reflejan las reducciones en el coste unitario del producto, servicio o procesos de todo tipo que se producen como consecuencia de mayores volúmenes de actividad por periodo. Presentan impedimentos porque fuerzan al pretendiente a operar a gran escala, con el riesgo de una inmediata reacción por parte de los competidores existentes, o a ingresar a pequeña escala aceptando una desventaja inicial en coste.

En la contratación del profesorado, pueden tener lugar en grandes centros acreditados o universidades. También los procesos de generación de contenidos pueden beneficiarse de las economías de escala en la producción, sobre todo en el caso de grandes repositorios de objetos de aprendizaje, tal y como se tratará al estudiar la estrategia competitiva de liderazgo en costes.

- Necesidades de capital

La necesidad de fuertes volúmenes de capital constituye una barrera de entrada, lo cual supone una ventaja para los competidores existentes.

El hecho de que el e-learning se caracterice por la realización de la formación a través de las TIC determina la importancia relativa de la inversión tecnológica y del uso de las mismas, de manera que se produce un desplazamiento de recursos, desde los elementos tradicionales basados en la infraestructura física hacia los bienes tecnológicos. Además, este gasto inicial está sometido a una obsolescencia muy rápida, lo cual genera un elevado nivel de riesgo para este tipo de inversiones.

Estos elevados costes iniciales en teoría se compensan por unos menores costes de personal y porque el alto gasto de desarrollo de curso se amortiza en las sucesivas repeticiones del mismo.

Este modelo impide la entrada de empresas que no dispongan de importantes presupuestos en generación de contenidos y de una cuota de mercado (número de matrículas) suficientemente grande para poder amortizar la inversión inicial. 
Una manera de reducir los costes asociados a las TIC es el fomento de los procesos de innovación tecnológica, por la necesidad de adaptar los estándares tecnológicos a las particularidades de los procesos formativos y de actualizar y mejorar continuamente la funcionalidad de los diferentes componentes del sistema.

\section{Productos o servicios sustitutivos}

Cabe hacer notar que la educación virtual puede también ser observada como un complemento de la educación presencial ya adquirida por un estudiante que desea complementar sus estudios o perfeccionarse.

En comparación con la educación presencial, la actitud del cliente hacia la educación virtual es menos favorable, debido a un problema de cultura (a veces, subestimada) y disciplina que ha significado que exista una mayor lealtad hacia los servicios tradicionales de las Universidades e Institutos, pero sólo en la medida en que éstos tengan un respaldo en cuanto a experiencia y a logros concretos en materia de educación.

Desde una perspectiva de sustitutos, la realidad es que no es la educación presencial el sustituto de la virtual, sino por el contrario, es la educación virtual la que se ha requerido como un muy buen sustituto de la educación presencial y lo será cada vez más en la medida que mejore la tecnología que soporta al e-learning así como el grado de alfabetización tecnológica de la población.

\section{Negociación con los agentes frontera}

Llamaremos agente frontera a cualquier actor con cierta capacidad de influencia sobre el sector que se estudia, en este caso, el e-learning en la Educación Superior. El poder negociador de las Universidades con los distintos agentes frontera que participan en su entorno (proveedores, clientes y Gobierno) difiere en función del actor.

En primer lugar, el poder negociador de los proveedores educativos es reducido puesto que las Universidades tienen capacidad para abarcar casi todo el sistema de valor completo. Sin embargo, también pueden ser considerados como proveedores los profesores, en relación a los cuales habría que subrayar que su poder negociador depende de los siguientes factores:

- Importancia estratégica del producto: adquiere importancia en cuanto el curso quiere tener cierto nivel o se pretende una diferenciación, ya que se precisa un material didáctico y profesorado cualificado en materias específicas difícilmente sustituibles.

- Concentración: suelen estar agrupados o relacionados con diversos tipos de asociaciones o entidades.

- Amenaza de integración hacia delante: existe la amenaza en el caso de que pertenezcan a asociaciones. Tienen como barrera la imagen de marca y la necesidad de capital.

- Peso de su coste en el producto: muy importante. 
En lo que respecta a los clientes, su poder negociador en formación reglada es escaso, como también lo es el de la Universidad al estar aquella regulada por el Estado. Sin embargo, en la formación no reglada, los estudiantes pueden presionar para que la Universidad baje los precios, bajo la amenaza de irse a la competencia o adquirir un producto o servicio sustitutivo.

Finalmente, el papel del Gobierno es grande puesto que tiene capacidad de influir directamente en el comportamiento del sector, favoreciendo la competencia por la financiación pública y disciplinando el mercado a través de la regulación.

\section{Segmentación}

La segmentación del mercado consiste en la definición de grupos de usuarios con necesidades razonablemente homogéneas, de tal forma que los clientes (estudiantes) de un mismo segmento del mercado en cuestión presentan necesidades que pueden ser satisfechas por el mismo producto o servicio.

Los criterios de segmentación dependen enormemente de las características del estudio que se desee llevar a cabo. En este artículo se ha adoptado una segmentación multicriterio basada tanto en el tipo de formación como en los rangos de edad del mercado objetivo.

En cuanto al tipo de formación, con el fin de que la clasificación escogida resulta válida tanto para la situación actual como para la marcada por las directrices del Espacio Europeo de la Educación Superior, se han considerado tres grandes segmentos:

- Formación de grado: estudiantes cuyo objetivo es obtener una licenciatura o diplomatura universitarias.

- Formación de postgrado: estudiantes, con una titulación de grado, que desean alcanzar el máximo grado académico profundizando sus estudios en alguna disciplina concreta.

- Formación continua: orientada a la adquisición de conocimientos, habilidades y competencias con el fin de adaptarse y posicionarse en el mercado laboral.

En general, puede observarse un predominio de la formación de grado frente a las otras dos en la mayor parte de los países europeos. Esta hegemonía de la formación de grado también se mantiene al comparar la formación virtual y la formación presencial.

Teniendo en cuenta los datos anteriores, y en una primera aproximación, podría parecer que las universidades tradicionales y las universidades virtuales compiten por el mismo mercado: la formación de grado. Por ello, es necesario analizar la situación también desde otra perspectiva que pueda arrojar mayor información sobre los segmentos del mercado.

Si nos centramos en el caso de la formación de grado y estudiamos el segmento por tramos de edad, vemos que en el caso de las universidades tradicionales, la mayor parte de los estudiantes se encuentra comprendida entre los 18 y los 24 años de edad. Este hecho contrasta con el hecho de que 
las Universidades Virtuales se centran más en la población de entre 25 y 40 años para el mismo tipo de formación.

Respecto al crecimiento del sector, aunque no han podido encontrarse datos que demuestren explícitamente, la evolución cuantitativa del e-learning en la Educación Superior, sí que se dispone de información suficiente para asegurar que se encuentra en la etapa de crecimiento de su ciclo de vida. Los indicios que justifican esta información son los siguientes:

- Aumento de la demanda universitaria debido, fundamentalmente, al incremento de la formación continua y la formación de personas adultas.

- Aumento de la penetración del Internet en la Sociedad.

- Aumento de las iniciativas de internacionalización y regionalización de la Educación Superior

- El número de Universidades que disponen de un campus virtual ha incrementando en los últimos años

\section{Cadena de valor}

El estudio detallado de la cadena de valor para cada institución y la optimización de las actividades que la constituyen, permite a dicha institución identificar las potenciales fuentes de ventaja competitiva. La cadena de valor de e-learning, a grandes rasgos, es la que se muestra en la Figura 3:

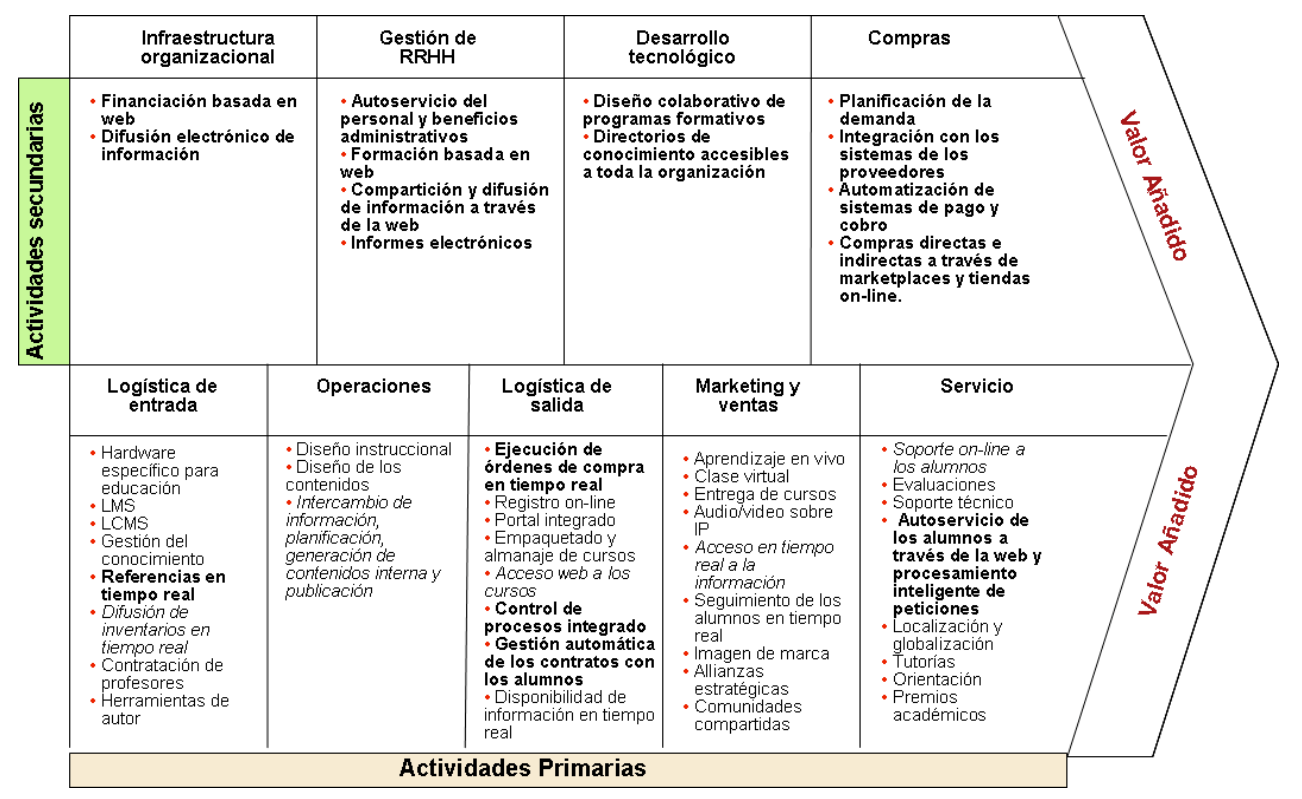

Figura 3. Cadena de valor del e-learning.

Fuente: elaboración propia

Teniendo esto e cuenta y a la hora de adaptar la cadena de valor de una universidad genérica para incorporar, a nivel institucional, el e-learninig como herramienta competitiva, nos parece necesario formular las siguientes propuestas en cada una de las grandes áreas de la cadena de valor: 
1) Investigación

Consideramos necesario potenciar las siguientes líneas de investigación:

a) Accesibilidad y usabilidad en plataformas de e-learning y su aplicación al campus virtual de la universidad.

b) Integración de nuevas tecnologías en plataformas de e-learning.

c) Evaluación de plataformas y contenidos.

d) Influencia del e-learning en la estrategia competitiva de las Universidades.

e) Aspectos legales de las TIC y del e-learning.

f) Desarrollo de estándares de calidad.

2) Docencia

a) Considerar las competencias TIC en el desarrollo profesional del PAS y del PDI.

b) Desarrollar de sistemas de evaluación y potenciación de la calidad del e-learning.

c) Adoptar estándares que favorezcan la reutilización de los contenidos de repositorios, tanto propios como ajenos.

d) Diseñar de un plan de incentivos adecuado.

3) Marketing y ventas

a) Diseñar una estrategia de marketing adecuada al e-learning

b) Establecer una oferta atractiva y ágil.

c) Optimizar el posicionamiento en buscadores.

4) Diseño de materiales educativos

a) Calidad de los contenidos.

b) Control de calidad en los contenidos.

c) Selección de profesorado que elabore los materiales.

d) Elección adecuada del software utilizado.

e) Creación de un grupo de revisores pedagógicos.

5) Servicios educativos 

a) Adopción de la filosofía CRM al entorno universitario.
b) Establecimiento de un servicio de atención al alumno.
c) Acceso a los recursos de una biblioteca digital.

6) Gestión de la institución

a) Adoptar un sistema de gestión para poder incorporar el e-learning en la actividad universitaria.

b) Mejorar la infraestructura TIC, sobre todo en seguridad y gestión basada en nuevas tecnologías que soporten aplicaciones como el pago on-line.

7) Gestión de recursos humanos

a) Formación específica del PAS y el PDI en e-learning

b) Creación de nuevas figuras adecuadas al entorno e-learning

8) Gestión académica

a) Evolucionar hacia una ventanilla on-line única para el alumno.

b) Incorporar los pagos on-line con el fin de abarcar todo el ciclo de producto.

9) Servicios de infraestructura de la Universidad

a) Dotar a las infraestructuras de los medios suficientes para un servicio de alta calidad.

b) Acercar el producto al usuario, sobre todo en el ámbito internacional.

c) Dimensionar adecuadamente los servidores para el servicio que se oferte.

d) Garantizar el servicio $365 \times 24 \times 7$.

\section{Estructura de costes}

En aras a facilitar su análisis, agruparemos los costes en tres grandes áreas: producción de materiales, realización de cursos y costes de gestión e infraestructura. Por otra parte, además de los costes propiamente dichos, es necesario incluir en el estudio una serie de factores que influyen en el comportamiento de los costes a lo largo del tiempo, entre los que destacan los siguientes:

- Duración del programa: un curso más largo permite una mejor distribución de los costes fijos y, por tanto, implica un menor coste fijo unitario por estudiante. 
- Repetición: los programas o los componentes individuales de cada curso pueden ser fácilmente reproducidos sin incurrir en costes adicionales.

- Duplicación: sin añadir costes de producción, los cursos y programas puede ser duplicados. Este elemento presenta esencialmente el mismo efecto que el manifestado en la duración del curso (mejor base de reparto de los costes fijos y menores costes unitarios por estudiante).

- Número de estudiantes: el aumento en el número de estudiantes permite asumir un claro efecto de escala y, consecuentemente, reducir el coste unitario por estudiante. Adicionalmente, y dado el valor relativamente reducido de los costes variables, un número de estudiantes adicional provocará un incremento muy reducido de los costes totales, potenciando la reducción de los costes totales medios.

- Punto de equilibrio: es el número de matrículas a partir del cual el programa de formación on-line resulta rentable (ver Figura 4).

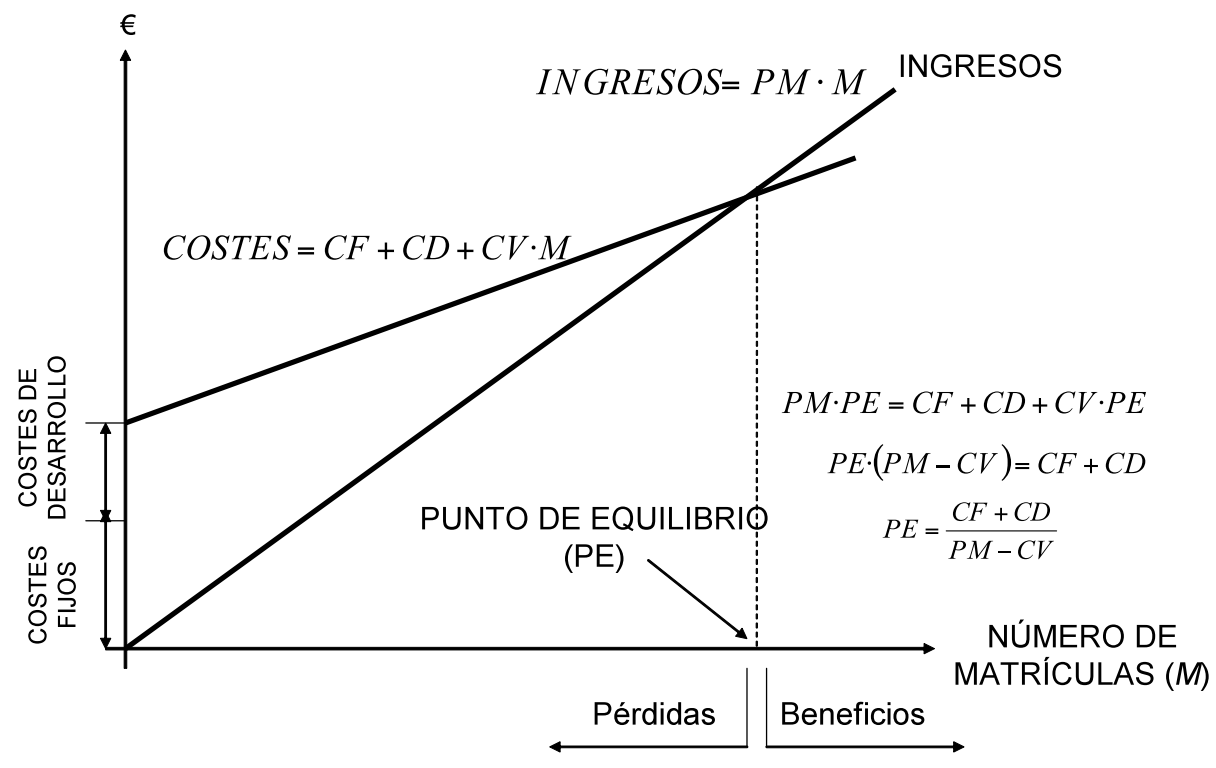

Figura 4. Análisis gráfico del punto de equilibrio.

Fuente: elaboración propia.

La distinción y clasificación de las distintas partidas de costes en costes fijos y costes variables (ver Figura 5) permite identificar que entre los primeros destacan los derivados del trabajo especializado y de la platforma tecnológica que, en su mayoría, se engloban en la generación de los contenidos, mientras que entre los variables, destaca el soporte docente on-line. 


\begin{tabular}{|l|c|c|}
\hline \multicolumn{1}{|c|}{ Partidas } & Coste fijo & Coste variable \\
\hline Expertos en la materia & $\mathrm{X}$ & \\
\hline Diseño instruccional & $\mathrm{X}$ & \\
\hline Especialista en Internet & $\mathrm{X}$ & \\
\hline Diseño de interfaces & $\mathrm{X}$ & \\
\hline Marketing & $\mathrm{X}$ & \\
\hline Servidor & $\mathrm{X}$ & \\
\hline Biblioteca & $\mathrm{X}$ & \\
\hline Derechos de propiedad de materiales y programas & $\mathrm{X}$ & \\
\hline Profesores internacionales & $\mathrm{X}$ & $\mathrm{X}$ \\
\hline Tutores docentes & & $\mathrm{X}$ \\
\hline Servicios de administración & & $\mathrm{X}$ \\
\hline Canon de la Universidad & & \\
\hline
\end{tabular}

Figura 5. Partidas de costes asociadas al e-learning en función de su categoría.

Fuente: adaptado de Castillo (2004).

\section{SISTEMA DE VALOR}

La cadena de valor también puede aplicarse para realzar las relaciones entre la organización y sus proveedores y entonces recibe el nombre de sistema de valor, que para el caso del e-learning puede verse en la Figura 6. Tanto la cadena de valor (a nivel interno) como el sistema de valor (a nivel externo), tienen como propósito inicial analizar las operaciones de la corporación para poder incrementar la eficiencia, efectividad y competitividad.

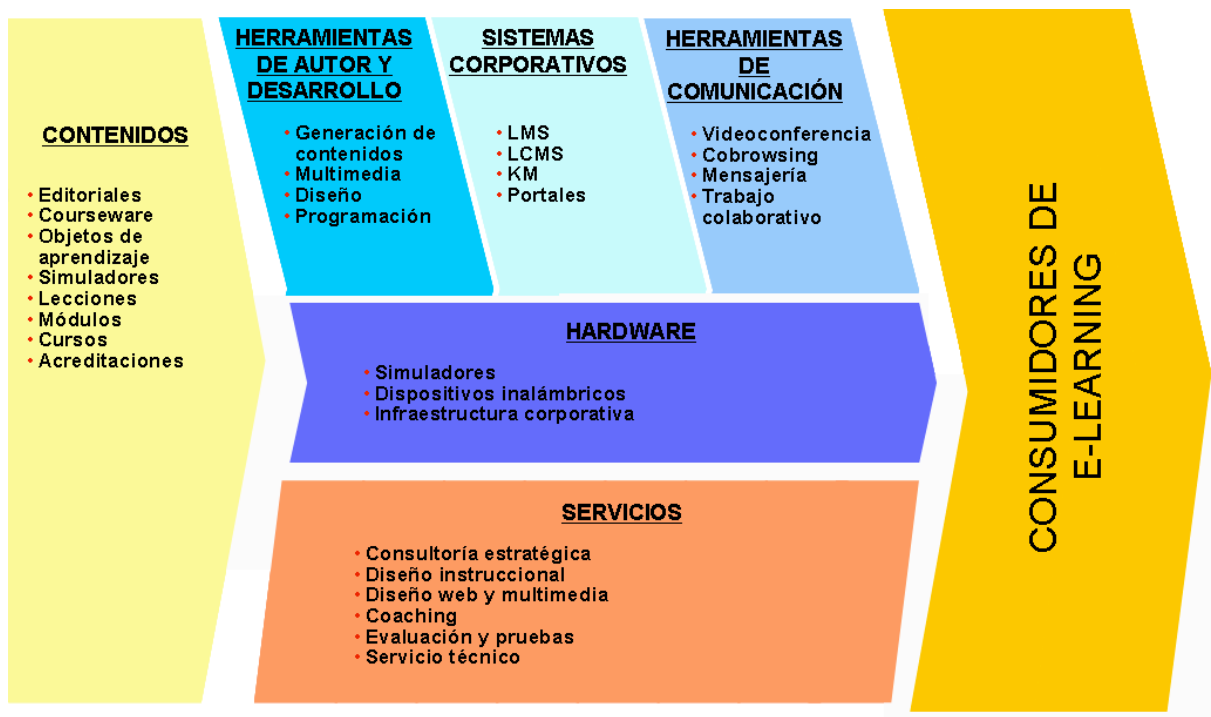

Figura 6. Sistema de valor del e-learning.

Fuente: adaptado de Elloumi (2004). 
Por supuesto, existe la posibilidad de concentrar toda esta provisión en una única entidad que denominaremos proveedor integral, como es el caso de las Universidades. Esto, en principio, les permite competir en el mercado sin ningún tipo de atadura o acuerdo con alguna otra organización.

Sin embargo, en algunas ocasiones resulta frecuente encontrar alianzas estratégicas entre universidades o bien entre universidad y empresa. La opción de competir sola o en compañía dependerá, en todo caso, de la estrategia institucional.

En general, existen tres tipos de alianzas estratégicas entre Universidades y proveedores educativos y que tienen sentido en el caso de una universidad genérica:

- Intermediario

La intermediación se basa en que una entidad pone en contacto a otra con el cliente final. Una posibilidad bastante común es la de un intermediario que vende los cursos de una o varias universidades aprovechando el prestigio y la imagen de marca de éstas. Generalmente, la empresa se dedica a los aspectos más relacionados con el marketing y las instituciones educativas se encargan del resto. Este modelo de negocio concede una gran importancia al intermediario y eso conlleva un elevado riesgo para las instituciones educativas que por una mala gestión del intermediario podrían ver comprometido su prestigio.

Un ejemplo de proveedor educativo que sigue este modelo es la OLA (Open Learning Australia), actualmente llamada Open Universities Australia (http://www.open.edu.au). Esta empresa se creó a finales de 1992 y actúa como intermediario en el sector de la Educación Superior australiana. Esta empresa es una asociación de siete universidades australianas y, desde que se fundara en 1993, ha acumulado alrededor de 100.000 matrículas. Los certificados que emite esta organización tienen la misma validez que los que obtienen los estudiantes que acuden a las universidades que forman parte de la asociación.

- Alianzas para ofrecer certificados profesionales

Existen empresas que emiten los llamados certificados profesionales, entre las que se encuentran Cisco, Microsoft, Novell, Oracle o el PMI (Project Management Institute). Una posibilidad para aumentar el valor de la oferta del proveedor y de la institución es integrar estos certificados profesionales en la oferta de títulos propios de la Universidad.

Merece la pena nombrar, el caso de la Universidad Politécnica de Valencia, en la que uno de los ejemplos más representativos es el Máster en Redes Corporativas e Integración de Sistemas que conduce a sus alumnos a una doble titulación: el CCNA o CCNP de Cisco, que caduca a los dos años; $y$, un título propio de la Universidad, que carece de fecha de caducidad.

- Alianzas para formación continua 
Recientemente, se están fomentando los acuerdos con empresas para la formación continua de trabajadores. Uno ejemplo de iniciativas en este sentido, de nuevo en la Universidad Politécnica de Valencia es el Master en Asesoría Financiera Europea que, recientemente, se ha adaptado a las necesidades particulares de los empleados de Bancaja.

\section{ESTRATEGIAS COMPETITIVAS}

Una vez que se ha definido el negocio y analizada la información recogida en los apartados anteriores, el paso siguiente será establecer con qué estrategia se competirá en el sector. Porter identificó dos estrategias competitivas genéricas: el liderazgo en costes y la diferenciación. Otra opción es la estrategia de nicho, que introduce en las dos opciones anteriores el concepto de ámbito de la estrategia para medir el alcance de la misma, de tal forma que la estrategia de nicho consiste en centrarse en un segmento del mercado, producto o servicio muy concreto y en el que se dispone de algún tipo de ventaja competitiva derivada de un liderazgo en costes o de una diferenciación derivadas, por ejemplo, del dominio de una cierta tecnología.

El liderazgo en costes, genéricamente, consiste en conseguir unos costes de producción más bajos que los competidores en un producto o servicio indiferenciado. En el e-learning, algunas estrategias útiles son la limitación de la oferta de cursos a sectores muy específicos, la reducción de la complejidad de los procesos de diseño y producción de material formativo o la disminución del servicio de atención a los estudiantes. Todo ello, complementado un estricto control de costes. Tampoco hay que olvidar los beneficios de las organizaciones virtuales que, entre otras cosas, permiten desarrollar una actividad de negocio sin necesidad de disponer de instalaciones físicas, ahorrándose los gastos y la inversión que ello supone. Un ejemplo de institución que ha apostado por una estrategia de liderazgo en costes en la University of Phoenix (UoP) contrando profesores a tiempo parcial y dedicándolos, única y exclusivamente, a labores de docencia y no otras tareas también propias del personal docente como la investigación y la gestión académica. La UoP también trata de mantener los costes controlados minimizando sus necesidades de infraestructura, por ejemplo, proporcionando multitud de recursos bibliográficos a través de Internet en lugar de disponer de una biblioteca propia.

Por tanto, uno de los puntos clave para conseguir el liderazgo en costes es la optimización del proceso de generación de contenidos. En este sentido, se aconseja la creación de grandes repositorios de objetos de aprendizaje que permitan, por otra parte, aprovecharse de los efectos de las economías de escala en la producción de cursos. Un repositorio de contenidos es un gran almacen de objetos de aprendizaje, es decir, de elementos modulares en los que se empaquetan los contenidos y que permiten la construcción de lecciones, cursos y, en general, cualquier tipo de elemento formativo, a partir del ensamblado "bajo demanda" de dichos objetos de aprendizaje (ver Figura 8): 


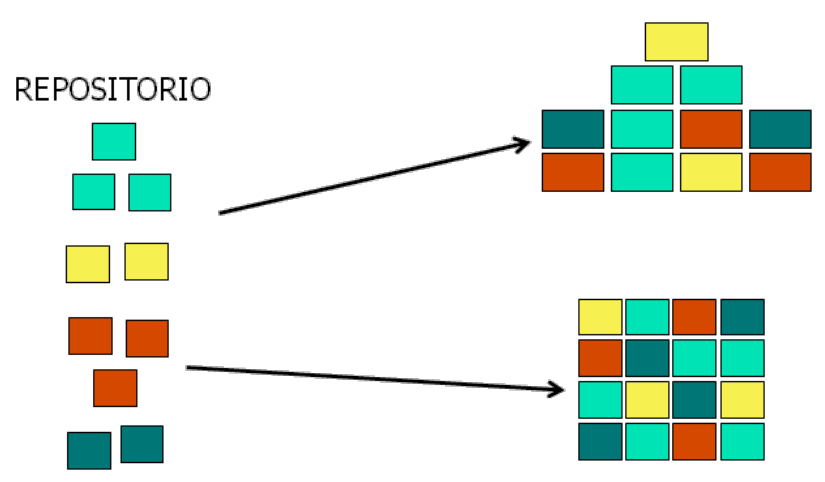

Figura 8. Construcción de cursos a partir de un repositorio digital

Fuente: elaboración propia.

El impacto de un repositorio digital en la estrategia de liderazgo en costes puede verse fácilmente si consideramos el análisis del punto de equilibrio. Como muestra la Figura 9, un repositorio digital se podría traducir en la reducción de los costes fijos asociados a la generación de contenidos, habida cuenta de la posibilidad de la reutilización de los mismos, y, por tanto, en un desplazamiento del punto de equilibrio hacia la izquierda, con la consiguiente maximización de la zona de beneficios que ello supone.

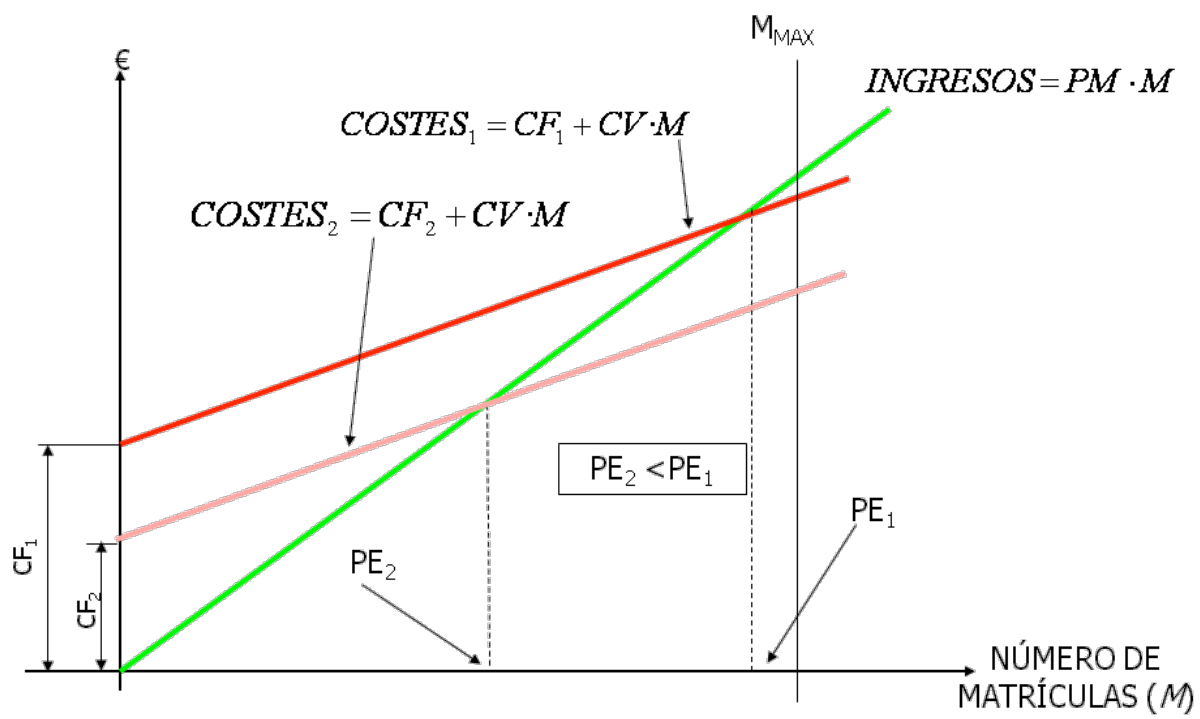

Figura 9. Aumento de la rentabilidad por efecto de un repositorio digital.

Fuente: elaboración propia.

En cuanto a la diferenciación, consiste en conseguir que el producto o servicio tenga, al menos, una característica única que lo distinga de los productos o servicios de la competencia y de modo que los clientes perciban un mayor valor en él. La esencia de la diferenciación en el e-learning es que, en general, un curso en sí mismono proporciona ninguna ventaja competitiva en tanto en cuanto 
cualquier organización puede duplicarlo, bien comprándolo directamente del proveedor o creándolo ella misma. Sin embargo, cuando al curso se le añaden los servicios prestados por profesores, tutores, etc., se establece un mayor vínculo entre los estudiantes y la organización educativa que se traduce en una ventaja competitiva en cuanto a soporte, servicio, excelencia y calidad. Precisamente, la calidad es el principal factor diferenciador en este sector para la creación de una imagen de prestigio. Esta calidad debe entenderse en dos sentidos: calidad de los contenidos y calidad del servicio.

\section{LÍNEAS DE INVESTIGACIÓN FUTURAS}

En las cuestiones abordadas en este trabajo de investigación, debido a la utilización de una estrategia de indicios, podría haberse caído en una visión demasiado simplista en algunas ocasiones. Esto demanda la construcción de un modelo que incorpore, en primer lugar, los factores relevantes en el campo de análisis y, en segundo lugar, que explore las relaciones entre dichos factores. Ello conducirá a explicar satisfactoriamente la contribución de cada factor a la estrategia de las Universidades.

La investigación adicional que pueda suscitarse a partir de esta tesis, debería incluir la medición y el grado de influencia de factores como la cultura institucional, el papel del personal clave, la experiencia internacional, factores geográficos, la inversión en TIC, etc., que quizá sean relevantes y cuyo papel debiera ser analizado y tomado en cuenta para investigaciones futuras. Todo ello permitirá la obtención de expresiones matemáticas más compleja de los factores descriptores de la rivalidad que han sido presentados aquí de un modo muy simplificado.

Finalmente, existe gran cantidad de estudios acerca de la Educación Superior presencial, por ser éste el formato tradicional. Sin embargo, la utilización del e-learning en Educación Superior como respuesta a la internacionalización de la misma, está mucho menos analizado y, además, carece de sustento empírico por la relativa juventud de este campo de análisis. Por tanto, existen aquí otras líneas futuras de investigación.

\section{CONCLUSIONES}

En los últimos años la Educación Superior se ha visto envuelta en un proceso de cambio como consecuencia de la internacionalización y la evolución hacia modelos más orientados a mercado que ha obligado a la Universidades a adoptar técnicas y estrategias propias del mundo empresarial, manejando conceptos típicos del mundo de los negocios.

En estas condiciones el secreto del éxito está en desarrollar un modelo de negocio que no solamente se adapte mejor a las características propias de la instutición y del entorno que la rodea forteleciendo aquellos aspectos sobre los que descansa su ventaja competitiva, sino que, además, erosione los modelos de negocio del resto de competidores. Con el fin de facilitar este análisis, el presente artículo propone un proceso sistemático de diseño de modelos de negocio, haciendo especial hincapié en los puntos críticos a la hora de elaborarlo. 
ROLDÁN, David; HERVÁS, Antonio (2008) «E-learning como estrategia de internacionalización de la educación superior» [artículo en línea]. EDUTEC, Revista Electrónica de Tecnología Educativa. Núm. 27/ Noviembre 2008. [Fecha de consulta: $\mathrm{dd} / \mathrm{mm} / \mathrm{aa}$ ].

http://edutec.rediris.es/revelec2/revelec27/

ISSN 1135-9250. 